\title{
Immune Checkpoint Inhibitors in Triple Negative Breast Cancer
}

\author{
Nibash Budhathoki ${ }^{1}$, Ajay Dhakal ${ }^{2 *}$ and Mateusz Opyrchal ${ }^{2}$ \\ ${ }^{1}$ Department of Medicine, Interfaith Medical Center, USA \\ ${ }^{2}$ Department of Medicine, Roswell Park Cancer Institute, USA
}

*Corresponding author: Ajay Dhakal, Clinical Fellow, Department of Medicine, Roswell Park Cancer Institute, University at Buffalo, Elm and Carlton Streets, 14263, Buffalo, New York, USA

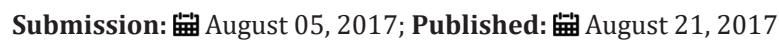

\begin{abstract}
Keywords: Immune Checkpoint Inhibitors; Triple Negative Breast Cancer; Breast Cancer; PD1; PD-L1, Pembrolizumab; Nivolumab; Atezolizumab
Abbreviations: CTLA4: Cytotoxic T Lymphocyte Antigen 4; PD1: Programmed Death 1; BMS: Bristol Myers Squibb; TIL: Tumor Infiltrating Lymphocytes; TNBC: Triple Negative Breast Cancer; IHC: Immunohistochemistry; CR: Complete Response; PR: Partial Response; SD: Stable Disease; PFS: Progression Free Survival; IC: Immune Cells; ORR: Overall Response Rate; pCR: Pathological Complete Response; HR: Hormone Receptor
\end{abstract}

\section{Introduction}

The roleofimmune system in treating canceris beingincreasingly seen as important [1]. Cancer cells have oncogenic mutations and express neo-antigens that make them distinct from normal cells thus prone to immune recognition. However, cells of innate and adaptive immune system which recognize and destroy these cells are held in check by various molecular pathways called "immune checkpoints" [2]. Attempts have been made to block these immune checkpoints to develop anticancer immunotherapies. Blockage of Cytotoxic T Lymphocyte Antigen 4 (CTLA4) and Programmed Death 1 (PD1), the first 2 checkpoint receptors to be discovered, has been studied widely in various cancers. Bristol Myers Squibb (BMS)'s ipilimumab, a monoclonal antibody against CTLA4, was efficacious and produced a durable response against melanoma. It was approved as the standard treatment for melanoma [3-5]. BMS's nivolumab, Merck's pembrolizumab, and Roche's atezolizumab are monoclonal antibodies against PD1 (nivolumab, pembrolizumab) or PD Ligand 1(PD-L1) (atezolizumab). They are efficacious in various malignancies including melanoma, kidney cancer, nonsmall cell lung cancer, urothelial cancer, and colon cancer [6-17]. PD-L1 expression has been suggested as a predictive biomarker for these antibodies but due to lack of a single standardized assay for measuring the expression of these surface proteins and lack of consistency in trial design across different antibodies, data supporting the predictive value of PD-L1 expression are not uniform [18].

\section{PD1/PD-L1 in Breast Cancer}

Ghebeh et al. published data on PD-L1 expression in breast cancer in [19]. They evaluated the presence of PD-L1 in either tumor cells or tumor infiltrating lymphocytes in primary breast cancers by immunohistochemistry (IHC) [19]. A large scale pooled analysis of almost 4000 primary breast cancer samples from 3 different studies also showed PD-L1 expression of $>1 \%$ of immune cells in $6 \%$ of tumors [20]. However, only $1.7 \%$ tumors expressed PD-L1 in $>1 \%$ of tumor cells. Subset analysis in the study showed that the breast cancers with core basal phenotype have higher PDL1 expression. In tumor cells, $8 \%$ of the samples had more than $1 \%$ of PD-L1 expression. In immune cells, it was seen in $18.5 \%$ of the samples (20). As core basal phenotype has a strong association with triple negative breast cancer (TNBC), this data suggested potential role of immune checkpoint inhibitors in TNBC [21]. Analysis of The Cancer Genome Atlas data demonstrated higher PDL1 mRNA expression in breast tissue specimens from patients with TNBC $(n=120)$ compared to patients with non-TNBC $(n=716)$ [22]. In the study, immuno-histochemical stain for PD-L1 expression was performed on a tissue microarray. It comprised of 105 tumors from early stage triple negative breast cancer patients that had not received neo-adjuvant chemotherapy. PD-L1 expression, defined as $>5 \%$ membranous staining, was identified in 20 (19\%) of the tumors in the study [22]. Additionally, TNBCs have the highest frequency of tumor infiltrating lymphocytes (TILs) [23]. The higher incidence of PD-L1 expressing cells (both immune and tumor cells) and the higher incidence of TILs in TNBC, has led to studies assessing efficacy of immune checkpoint inhibition in this type of breast cancer.

\section{Checkpoint Inhibition in Metastatic Triple Negative Breast Cancer}

KEYNOTE-012, a multicenter, nonrandomized phase Ib trial of single-agent pembrolizumab was done among patients with 
advanced PD-L1-positive (expression in stroma of $\geq 1 \%$ of tumor cells by immunohistochemistry) TNBC, gastric cancer, urothelial cancer, and head and neck cancer [24]. Among 27 women with TNBC, the overall response rate was $18.5 \%$. Disease control rate, defined as complete response (CR), partial response (PR) or stable disease (SD) for at least 24 weeks, was seen in a $25 \%$ of patients. The 6-month progression free survival (PFS) was observed in $25 \%$ of patients. The median duration of response was not reached by the time of data analysis. The duration of response ranged from 15 to $>47.3$ weeks. Based on historical data, the median duration of disease control in metastatic TNBC with first line, second and third line standard cytotoxic chemotherapy was 12 weeks, 9 weeks and 4 weeks respectively [25]. This study showed a promising disease control rate with pembrolizumab with acceptable safety and tolerability profile in a heavily pretreated TNBC patient population [24].

Keynote 086 (cohort A), a phase II trial assessed the safety and the efficacy of pembrolizumab in previously treated metastatic TNBC regardless of PD-L1 expression. Primary outcome was overall response rate for up to 24 months. Sixty percent of the patients had PD-L1 positive tumors. After a median follow-up of 11.9 months among 170 patients, the overall response rate was $5 \%$ and the disease control rate was $8 \%$. The response rate was similar between PD-L1 positive and negative tumors. The median PFS and the overall survival (OS) were 2.0 month (95\% CI 1.9-2.0) and 8.9 months (95\% CI 7.2-11.2). Six months of PFS and OS were seen in $12 \%$ and $69 \%$ patients respectively [26]. Cohort B of KEYNOTE 086 assessed the safety and antitumor activity of pembrolizumab as first-line therapy for patients with PD-L1 positive metastatic TNBC.
After a median follow-up of 7 months in 52 enrolled patients, the overall response rate was $23 \%$, the median PFS was 2.1 months (95\% CI, 2.0-3.9); the estimated 6-month PFS rate was $29 \%$ and the median duration of response was 8.4 months [27]. Though full manuscripts are awaited for Keynote 086 studies, these presented data show promising antitumor activity and disease control in previously untreated metastatic PD-L1 positive TNBCs.

Schmid et al. evaluated atezolizumab in a cohort of 115 metastatic TNBC patients in a Phase Ia study (NCT01375842) for long-term clinical outcomes (CR, PR and SD for >=12 weeks) and biomarkers [28]. Baseline PD-L1 expression on tumor infiltrating immune cells (IC) was centrally scored as IC $0 / 1 / 2 / 3$ based on VENTANA SP142 assay. Enrollment was initially limited to TNBC patients with PD-L1 expression in $\geq 5 \%$ of tumor-infiltrating immune cells (IC2/3), and then opened to all TNBC patients regardless of PD-L1 status. Atezolizumab was well tolerated. The overall response rate in 1st line and subsequent line therapies was $26 \%$ and $11 \%$ respectively. The overall response rate (ORR) for PDL1 IC2/3 patients was $17 \%$ vs $8 \%$ in IC0/1. The median duration of response was 21.1 months. The overall survival rates in all patients at 1,2 , and 3 years were $41 \%, 22 \%$, and $22 \%$, respectively. This study showed that atezolizumab has acceptable safety profile and promising antitumor activity with durable responses in both 1st line and subsequent lines of therapy. Also, ORR was higher among tumors with high PD-L1 expressing immune cells. But this study and earlier mentioned Keynote 086 are early phase single arm studies. Phase III randomized studies are underway to establish the clinical benefit of these agents in metastatic TNBCs (Table 1).

Table 1: List of ongoing phase III clinical trials assessing checkpoint inhibitors in TNBC in metastatic and perioperative settings.

\begin{tabular}{|c|c|}
\hline Trial Number & Study Overview \\
\hline NCT02555657 & $\begin{array}{l}\text { A randomized open-label phase III study of single agent pembrolizumab versus single agent chemotherapy per physician's choice for } \\
\text { metastatic triple negative breast cancer }\end{array}$ \\
\hline NCT02819518 & $\begin{array}{l}\text { A randomized, double-blind, phase III study of pembrolizumab plus chemotherapy versus placebo plus chemotherapy for previously } \\
\text { untreated locally recurrent inoperable or metastatic triple negative breast cancer }\end{array}$ \\
\hline NCT03036488 & $\begin{array}{l}\text { A phase III, randomized, double-blind study to evaluate pembrolizumab plus chemotherapy versus placebo plus chemotherapy as } \\
\text { neo-adjuvant therapy and pembrolizumab versus placebo as adjuvant therapy for triple negative breast cancer }\end{array}$ \\
\hline NCT02954874 & $\begin{array}{l}\text { A randomized, phase III trial to evaluate the efficacy and safety of pembrolizumab as adjuvant therapy for triple negative breast } \\
\text { cancer with } \geq 1 \mathrm{~cm} \text { residual invasive cancer or positive lymph nodes (ypn+) after neo-adjuvant chemotherapy }\end{array}$ \\
\hline NCT03125902 & $\begin{array}{l}\text { A phase III, multicenter, randomized, double-blind, placebo-controlled study of atezolizumab in combination with paclitaxel compared } \\
\text { with placebo with paclitaxel for patients with previously untreated inoperable locally advanced or metastatic triple negative breast } \\
\text { cancer }\end{array}$ \\
\hline NCT02425891 & $\begin{array}{l}\text { A phase III, multicenter, randomized, placebo-controlled study of atezolizumab in combination with nab-paclitaxel compared with } \\
\text { placebo with nab-paclitaxel for patients with previously untreated metastatic triple negative breast cancer }\end{array}$ \\
\hline NCT03197935 & $\begin{array}{l}\text { A phase III randomized study to investigate the efficacy and safety of atezolizumab in combination with neo-adjuvant anthracycline/ } \\
\text { nab-paclitaxel-based chemotherapy compared with placebo and chemotherapy in patients with primary invasive triple negative } \\
\text { breast cancer }\end{array}$ \\
\hline NCT02620280 & $\begin{array}{l}\text { A phase III neo-adjuvant study of atezolizumab in triple negative locally advanced breast cancer undergoing treatment with nab- } \\
\text { paclitaxel and carboplatin }\end{array}$ \\
\hline
\end{tabular}




\section{Checkpoint Inhibition in Adjuvant/Neo-adjuvant Setting for Triple Negative Breast Cancer}

I-SPY 2 is a multi-center, phase 2, platform trial that evaluates novel neo-adjuvant therapies; the primary endpoint being the pathological complete response (pCR). The result from I-SPY 2 studying pembrolizumab plus standard neo-adjuvant therapy for high-risk breast cancer was presented at ASCO annual meeting 2017 [29]. Enrolled patients in the study had clinically or radiologically measurable disease in the breast after diagnostic biopsy, defined as $\geq 2.5 \mathrm{~cm}$ by exam or $\geq 2 \mathrm{~cm}$ by imaging; eligible patients also had to meet one of the following criteria: stage II/III, or T4/anyN/M0 (including clinical or pathologic inflammatory cancer or regional stage IV disease, where supraclavicular lymph nodes are the only sites of metastasis). The patients were randomized to either weekly paclitaxel x 12 or with pembrolizumab prior to surgery followed by doxorubicin/cyclophosphamide $\mathrm{x} 4$ cycles in the adjuvant setting. The study showed that the patients with locally advanced TNBC or hormone receptor (HR)-positive/HER2-negative breast cancer had an increase in the estimated pCR rate in pembrolizumab arm. In TNBC, there was nearly threefold increase in patients who achieved pCR (60\% vs $20 \%$ ). Similar benefit was seen in patients with HR-positive/HER2-negative breast cancer (34\% vs $13 \%$ ) and in all HER2-negative patients (46\% vs $16 \%$ ). The safety profile of pembrolizumab was consistent with that observed in previously reported studies across tumors. The results of this study show potential promise for the anti PD1 therapy in combination with cytotoxic chemotherapy in a neo-adjuvant setting in high risk locally advanced breast cancers especially in TNBC. Further studies are currently underway assessing the benefit of checkpoint inhibitors in TNBC in adjuvant/neo-adjuvant settings (Table 1).

\section{Conclusion}

Anti PD1 and anti PD-L1 therapies have shown to have antitumor activity and durable responses in metastatic breast cancer. The best efficacy has been seen in patients with TNBCs. Similarly, in neo-adjuvant setting, results are encouraging with the highest responses seen in TNBC. Pembrolizumab has shown to produce a 3 fold increase in the pathological CR when used in combination with weekly paclitaxel in high risk locally advanced TNBCs. It is unclear if PD1 expression or other biomarkers will be predictive for response to this class of agents; this is an area of intensive research. Pembrolizumab, nivolumab, atezolizumab, durvalumab, ipilimumab and tremelimumab are being tested in phase I/II studies as single agents or in combinations in patients with high risk or metastatic breast cancer. We will await the results of these studies to determine how this class of drugs can be best used to maximize the efficacy in breast cancer patients.

\section{Conflict of Interest}

\section{Ajay Dhakal MBBS and Nibash Budhathoki MBBS: None}

Mateusz Opyrchal MD, PhD: Has received research fund from Pfizer.

\section{References}

1. Schreiber RD, Old LJ, Smyth MJ (2011) Cancer immunoediting: integrating immunity's roles in cancer suppression and promotion. Science 331(6024): 1565-1570.

2. Topalian SL, Drake CG, Pardoll DM (2015) Immune checkpoint blockade: a common denominator approach to cancer therapy. Cancer Cell 27(4): 450-461.

3. Hodi FS, O'Day SJ, Mc Dermott DF, Weber RW, Sosman JA, et al. (2010) Improved survival with ipilimumab in patients with metastatic melanoma. N Engl J Med 363(8): 711-723.

4. Robert C, Thomas L, Bondarenko I, O Day S, Weber J, et al. (2011) Ipilimumab plus dacarbazine for previously untreated metastatic melanoma. N Engl J Med 364(26): 2517-2526.

5. Schadendorf D, Hodi FS, Robert C, Weber JS, Margolin K, et al. (2015) Pooled Analysis of Long-Term Survival Data From Phase II and Phase III Trials of Ipilimumab in Unresectable or Metastatic Melanoma. J Clin Oncol Off J Am Soc Clin Oncol 33(17): 1889-1894.

6. Balar AV, Galsky MD, Rosenberg JE, Powles T, Petrylak DP, et al. (2017) Atezolizumab as first-line treatment in cisplatin-ineligible patients with locally advanced and metastatic urothelial carcinoma: a single-arm, multicentre, phase 2 trial. Lancet Lond Engl 389(10064): 67-76.

7. Brahmer J, Reckamp KL, Baas P, Crinò L, Eberhardt WEE, et al. (2015) Nivolumab versus Docetaxel in Advanced Squamous-Cell Non-Small-Cell Lung Cancer. N Engl J Med 373(2): 123-135.

8. Carbone DP, Reck M, Paz-Ares L, Creelan B, Horn L, et al. (2017) FirstLine Nivolumab in Stage IV or Recurrent Non-Small-Cell Lung Cancer. N Engl J Med 376(25): 2415-2426.

9. Herbst RS, Baas P, Kim D-W, Felip E, Pérez-Gracia JL, et al. (2016) Pembrolizumab versus docetaxel for previously treated, PD-L1-positive, advanced non-small-cell lung cancer (KEYNOTE-010): a randomised controlled trial. Lancet Lond Engl 387(10027): 1540-1550.

10. Larkin J, Chiarion-Sileni V, Gonzalez R, Grob JJ, Cowey CL, et al. (2015) Combined Nivolumab and Ipilimumab or Monotherapy in Untreated Melanoma. N Engl J Med 373(1):23-34.

11. Le DT, Uram JN, Wang H, Bartlett BR, Kemberling H, et al. (2015) PD-1 Blockade in Tumors with Mismatch-Repair Deficiency. N Engl J Med 372(26): 2509-2520.

12. Motzer RJ, Escudier B, McDermott DF, George S, Hammers HJ, et al. (2015) Nivolumab versus Everolimus in Advanced Renal-Cell Carcinoma. N Engl J Med 373(19): 1803-1813.

13. Reck M, Rodríguez-Abreu D, Robinson AG, Hui R, Csőszi T, et al. (2016) Pembrolizumab versus Chemotherapy for PD-L1-Positive Non-SmallCell Lung Cancer. N Engl J Med 375(19): 1823-1833.

14. Robert C, Long GV, Brady B, Dutriaux C, Maio M, et al. (2015) Nivolumab in previously untreated melanoma without BRAF mutation. $\mathrm{N}$ Engl J Med 372(4): 320-330.

15. Robert C, Schachter J, Long GV, Arance A, Grob JJ, et al. (2015) Pembrolizumab versus Ipilimumab in Advanced Melanoma. N Engl J Med 372(26): 2521-2532.

16. Rosenberg JE, Hoffman-Censits J, Powles T, van der Heijden MS, Balar $\mathrm{AV}$, et al. (2016) Atezolizumab in patients with locally advanced and metastatic urothelial carcinoma who have progressed following treatment with platinum-based chemotherapy: a single-arm, multicentre, phase 2 trial. Lancet Lond Engl 387(10031): 1909-1920.

17. Wolchok JD, Kluger H, Callahan MK, Postow MA, Rizvi NA, et al. (2013) Nivolumab plus ipilimumab in advanced melanoma. N Engl J Med 369(2): 122-133

18. Hansen AR, Siu LL (2016) PD-L1 Testing in Cancer: Challenges in Companion Diagnostic Development. JAMA Oncol 2(1): 15-16.

19. Ghebeh H, Mohammed S, Al-Omair A, Qattan A, Lehe C, et al. (2006) The B7-H1 (PD-L1) T lymphocyte-inhibitory molecule is expressed in breast 
cancer patients with infiltrating ductal carcinoma: correlation with important high-risk prognostic factors. Neoplasia N Y N 8(3): 190-198.

20. Ali HR, Glont S-E, Blows FM, Provenzano E, Dawson S-J, et al. (2015) PD-L1 protein expression in breast cancer is rare, enriched in basal-like tumours and associated with infiltrating lymphocytes. Ann Oncol Off J Eur Soc Med Oncol 26(7): 1488-1493.

21. Bertucci F, Finetti P, Cervera N, Esterni B, Hermitte F, et al. (2008) How basal are triple-negative breast cancers? Int J Cancer 123(1): 236-240.

22. Mittendorf EA, Philips AV, Meric-Bernstam F, Qiao N, Wu Y, et al. (2014) PD-L1 expression in triple-negative breast cancer. Cancer Immunol Res 2(4): 361-370

23. Stanton SE, Adams S, Disis ML (2016) Variation in the Incidence and Magnitude of Tumor-Infiltrating Lymphocytes in Breast Cancer Subtypes: A Systematic Review. JAMA Oncol 2(10): 1354-1360.

24. Nanda R, Chow LQM, Dees EC, Berger R, Gupta S, et al. (2016) Pembrolizumab in Patients With Advanced Triple-Negative Breas Cancer: Phase Ib KEYNOTE-012 Study. J Clin Oncol Off J Am Soc Clin Oncol. 34(21): 2460-2467.
25. Kassam F, Enright K, Dent R, Dranitsaris G, Myers J, et al. (2009) Survival outcomes for patients with metastatic triple-negative breast cancer: implications for clinical practice and trial design. Clin Breast Cancer 9(1): 29-33.

26. Adams S, Schmid P, Rugo HS, Winer EP, Loirat D, et al. (2017) Phase 2 study of pembrolizumab (pembro) monotherapy for previously treated metastatic triple-negative breast cancer (mTNBC): KEYNOTE-086 cohort A. J Clin Oncol 35(15): 1008-1008.

27. Adams S, Loi S, Toppmeyer D, Cescon DW, De Laurentiis M, et al. (2017) Phase 2 study of pembrolizumab as first-line therapy for PD-L1-positive metastatic triple-negative breast cancer (mTNBC): Preliminary data from KEYNOTE-086 cohort B. J Clin Oncol 35(15): 1088-1088.

28. Schmid P, Cruz C, Braiteh FS, Eder JP, Tolaney S, et al. (2017) Atezolizumab in metastatic TNBC (mTNBC): Long-term clinical outcomes and biomarker analyses. Cancer Res 77(13): 2986-2986.

29. Nanda R, Liu MC, Yau C, Asare S, Hylton N, et al. (2017) Pembrolizumab plus standard neoadjuvant therapy for high-risk breast cancer (BC): Results from I-SPY 2. J Clin Oncol 35(15):506-506. 